\title{
Effect of fosmidomycin on metabolic and transcript profiles of the methylerythritol phosphate pathway in Plasmodium falciparum
}

\author{
María B Cassera ${ }^{*}$, Emilio F Merino**, Valnice J Peres, Emilia A Kimura, \\ Gerhard Wunderlich, Alejandro M Katzin ${ }^{+}$
}

\begin{abstract}
Departamento de Parasitologia, Instituto Ciências Biomédicas, Universidade de São Paulo, Av. Prof. Lineu Prestes 1374 05508-000 São Paulo, SP, Brasil *Department of Biochemistry, Yeshiva University, The Bronx, NY, US **Department of Medical Parasitology, NYU School of Medicine, New York, NY, US
\end{abstract}

In Plasmodium falciparum, the formation of isopentenyl diphosphate and dimethylallyl diphosphate, central intermediates in the biosynthesis of isoprenoids, occurs via the methylerythritol phosphate (MEP) pathway. Fosmidomycin is a specific inhibitor of the second enzyme of the MEP pathway, 1-deoxy-D-xylulose-5-phosphate reductoisomerase. We analyzed the effect of fosmidomycin on the levels of each intermediate and its metabolic requirement for the isoprenoid biosynthesis, such as dolichols and ubiquinones, throughout the intraerythrocytic cycle of $\mathrm{P}$. falciparum. The steady-state RNA levels of the MEP pathway-associated genes were quantified by real-time polymerase chain reaction and correlated with the related metabolite levels. Our results indicate that MEP pathway metabolite peak precede maximum transcript abundance during the intraerythrocytic cycle. Fosmidomycin-treatment resulted in a decrease of the intermediate levels in the MEP pathway as well as in ubiquinone and dolichol biosynthesis. The MEP pathway associated transcripts were modestly altered by the drug, indicating that the parasite is not strongly responsive at the transcriptional level. This is the first study that compares the effect of fosmidomycin on the metabolic and transcript profiles in P. falciparum, which has only the MEP pathway for isoprenoid biosynthesis.

Key words: Plasmodium falciparum - malaria - fosmidomycin - isoprenoid biosynthesis - real time polymerase chain reaction

Malaria is a leading cause of morbidity and mortality in the tropical regions, with 300 to 500 million clinical cases and 1.5 to 2.7 million deaths per year (Snow et al. 2005). With the availability of the complete genome sequence from Plasmodium falciparum, increasing attention has focused on transcript profiling and proteomic analyses of the parasite stages responsible for severe disease and pathogenesis in humans (Florens et al. 2002, Bozdech et al. 2003, Le Roch et al. 2003, Nirmalan et al. 2004, Llinas et al. 2006).

Two different biosynthetic routes are used to produce isopentenyl diphosphate (IPP) and dimethylallyl diphosphate (DMAPP) for isoprenoid biosynthesis including ubiquinones, dolichols and the prenylation of proteins (Sacchettini \& Poulter 1997, Sinensky 2000, Barkovich \& Liao 2001). In mammals, plants (cytoplasm), fungi, some bacteria and several protozoa, the isoprenic units are derived from the classical mevalonate pathway (Goldstein \& Brown 1990). In plastids of plants, several algae, eubacteria, cyanobacteria and apicomplexa

Financial support: Fapesp, CNPq, Pronex, European Commission (INCO-Dev, ICA4-CT-2001-10078). MBC and EFM were supported by Fapesp. EFM was a post-doctoral fellow in HA del Portillo's laboratory.

+Corresponding author: amkatzin@icb.usp.br

Received 1 March 2007

Accepted 14 May 2007 (apicoplast), the 2C-methyl-D-erythritol-4-phosphate (MEP) pathway produces IPP and DMAPP (Rohmer 1999). The MEP pathway starts with the condensation of pyruvate and glyceraldehyde-3-phosphate (GAP), which yields 1-deoxy-D-xylulose-5-phosphate (DOXP) catalyzed by DOXP synthase (DXS); for reviews see references (Lichtenthaler 1999, Rohmer 1999, Eisenreich et al. 2004, Rodriguez-Concepcion 2004). DOXP reductoisomerase (DXR) then catalyzes the intramolecular rearrangement and reduction of DOXP to MEP. The activity of this enzyme is specifically inhibited by fosmidomycin (Kuzuyama et al. 1998). Several subsequent reaction steps are necessary for the conversion of MEP to IPP and DMAPP (Fig. 1).

Discovery of the MEP pathway for isoprenoid biosynthesis in P. falciparum revealed several antimalarial drug targets (Jomaa et al. 1999). Jomaa and co-workers demonstrated that fosmidomycin and its derivate FR900098, are able to inhibit the growth of $P$. falciparum in culture and to cure mice infected with the related malaria parasite, P. vinckei (Jomaa et al. 1999). Recent field trials in humans have also demonstrated the effectiveness of fosmidomycin in the treatment of human malarial infections, but it has to be administered for more than four days when used alone (Missinou et al. 2002, Borrmann et al. 2005, 2006). Recently, biochemical and mass spectrometric analyses revealed that the MEP pathway is functionally active in all intraerythrocytic stages of $P$. falciparum (Cassera et al. 2004).

In this study we characterized the effect of fosmidomycin on the metabolic levels of each intermediate of the MEP pathway as well as dolichol and ubiquino- 


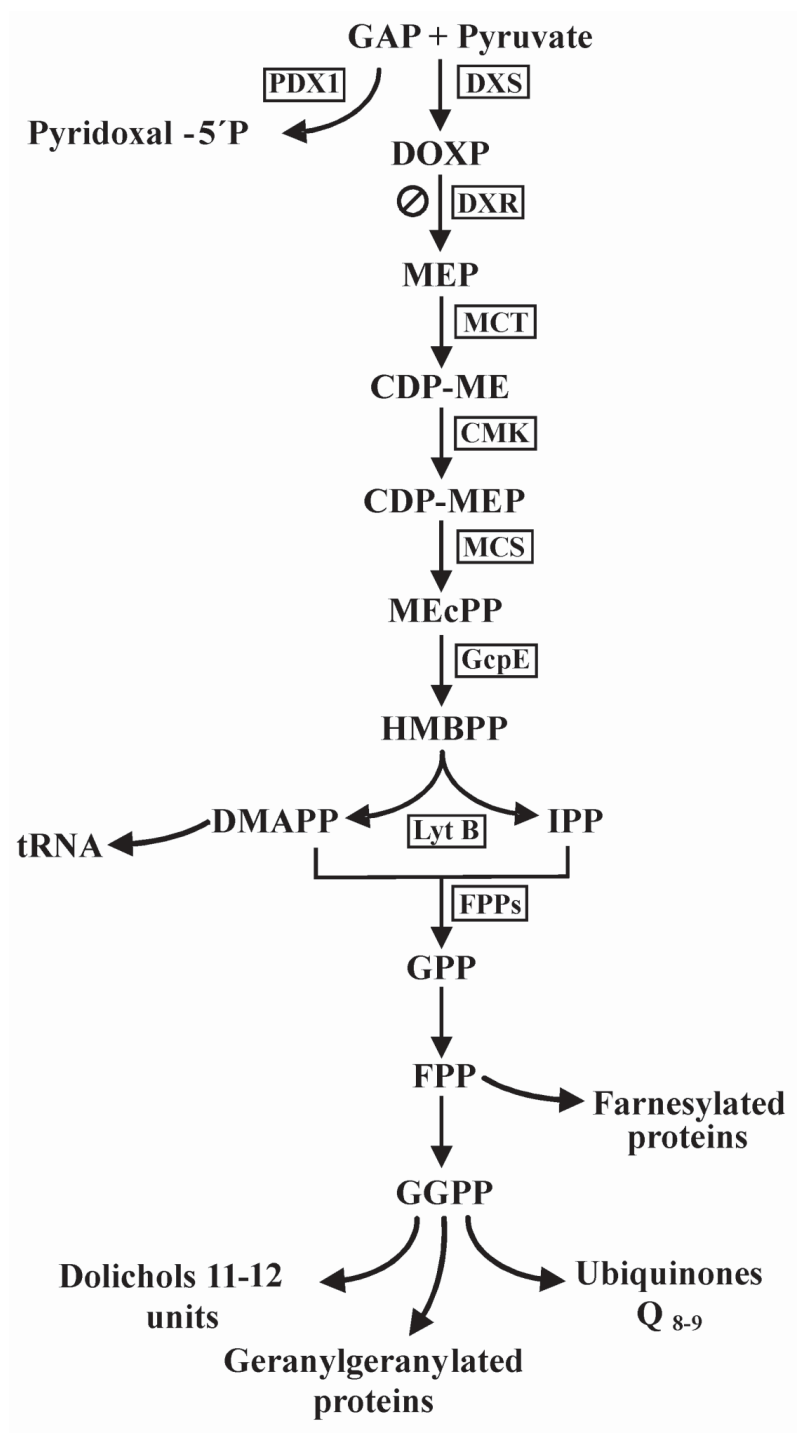

Fig. 1: isoprenoid biosynthesis pathway in Plasmodium falciparum. $\varnothing$ indicates the step that is inhibited by fosmidomycin. The following metabolites and enzymes are shown: PDX1, pyridoxal-5'-phosphate synthase; GAP, glyceraldehyde-3-phosphate; DOXP, 1-deoxy-D-xylulose-5-phosphate; DXS, DOXP synthase; MEP, 2C-methyl-D-erythritol4-phosphate; DXR, DOXP reductoisomerase; CDP-ME, 4-(cytidine-5diphospho)-2C-methyl-D-erythritol; MCT, 2C-methyl-D-erythritol-4-(cytidine-5-diphospho) transferase; CDP-MEP, 4-(cytidine-5-diphospho)-2Cmethyl-D-erythritol-2-phosphate; CMK, CDP-ME kinase; MEcPP, 2Cmethyl-D-erythritol-2,4-cyclodiphosphate; MCS, MEcPP synthase; HMBPP, 4-hydroxy-3-methyl-but-2-enyl pyrophosphate; GcpE, HMBPP synthase; IPP, isopentenyl diphosphate; DMAPP, dimethylallyl diphosphate; LytB, HMBPP reductase; GPP, geranyl diphosphate; FPP, farnesyl diphosphate; FPPs, FPP synthase; GGPP, geranylgeranyl diphosphate.

nes in ring, trophozoite and schizont stages of $P$. falciparum parasites and correlated these to the steadystate MEP enzyme transcript levels under drug pressure.

\section{MATERIALS AND METHODS}

Experimental design - Three characteristic developmental stages: ring ( $6 \mathrm{~h} \pm 4$ after reinvasion), trophozoite $(23 \mathrm{~h} \pm 4$ after reinvasion) and schizont $(36 \mathrm{~h} \pm 4$ after reinvasion) were chosen as representative for the intraerythrocytic cycle of $P$. falciparum. Two independent experiments using $\left[1-{ }^{14} \mathrm{C}\right]$ sodium acetate as a metabolic precursor were conducted to monitor each intermediate of the MEP pathway, dolichols and ubiquinones for each developmental stage. $\left[1-{ }^{14} \mathrm{C}\right]$ sodium acetate is well incorporated into the MEP pathway intermediates in P. falciparum (Cassera et al. 2004) instead of [1$\left.{ }^{14} \mathrm{C}\right]$ pyruvic acid or $\left[2-{ }^{14} \mathrm{C}\right]$ pyruvic acid, which is not incorporated by blood-stage $P$. falciparum (Cranmer et al. 1995, Elliott et al. 2001). Two cycles after sorbitol synchronization, cultures in ring, trophozoite or schizont stages with approximately $10 \%$ parasitemia, untreated or treated with $1 \mu \mathrm{M}$ fosmidomycin for $31 \mathrm{~h}$, were labeled with $6.25 \mu \mathrm{Ci} / \mathrm{ml}$ of $\left[1{ }^{14} \mathrm{C}\right]$ sodium acetate $(56$ $\mathrm{mCi} / \mathrm{mmol}$, Amersham Biosciences) in the last $17 \mathrm{~h}$, and recovered for analysis. Seventeen hours of exposure time is the minimum time to detect ${ }^{14} \mathrm{C}$ incorporation into the MEP pathway intermediates (Cassera et al. 2004). The parasites were isolated by treatment with $0.1 \%$ (w/v) saponin for $5 \mathrm{~min}$, followed by two washes with phosphate-buffered saline (PBS), pH 7.2, and stored in liquid $\mathrm{N}_{2}$ for subsequent HPLC analysis. Analyses of metabolites were accomplished by using $1 \times 10^{11}$ parasites of each stage obtained from treated or untreated synchronous cultures of $P$. falciparum. The same numbers of uninfected erythrocyte were analyzed in parallel. For transcript analysis, samples of RNA from three independent experiments were prepared using the same scheme of synchronization and treatment for the metabolic labeling described above. The $\mathrm{IC}_{50}$ value of fosmidomycin for the 3D7 strain is $1.25 \pm 0.05 \mu \mathrm{M}$ (Cassera et al. 2004). All experiments were performed with $1 \mu \mathrm{M}$ fosmidomycin during $48 \mathrm{~h}$ because under these conditions only a small percentage of parasites die and the length of the developmental cycle are not affected. The overall protein synthesis was controlled by quantification of TCA precipitates and by SDS-PAGE analysis at $48 \mathrm{~h}$. Protein synthesis was not affected in accordance to our earlier results (Cassera et al. 2004). Microscopic evaluation of Giemsa-stained thin smears was carried out very carefully to ensure that the parasites were at the same stage of development in control and fosmidomycin-treated cultures. Percent effect was determined as follows: [100 - (cpm or copy number in treated parasites $\times 100 / \mathrm{cpm}$ or copy number in untreated parasites)].

Parasite cultures - All experiments were performed with the $P$. falciparum 3D7 clone. Parasites were cultivated according to the method of Trager and Jensen (1976), modified by Kimura et al. (1996). Culture synchronization was carried out by two treatments with $5 \%$ (w/v) sorbitol solution in water (Lambros \& Vanderberg 1979).

RNA isolation, cDNA synthesis and real-time quantitative transcript analysis - Total RNA was prepared directly from $200 \mu \mathrm{l}$ of a saponin-treated cell pellet lysed in $2 \mathrm{ml} \mathrm{Trizol}{ }^{\circledR}$ (Invitrogen) and RNA was extracted according to the manufacturer's instructions. Subsequent cDNA synthesis for construction of the standard curves and quantitative real time PCR were performed with 5 $\mu \mathrm{g}$ total RNA from ring, trophozoite or schizont stages 
parasites, from three independent experiments untreated or treated with $1 \mu \mathrm{M}$ fosmidomycin for $48 \mathrm{~h}$. All RNAs were treated with DNAseI (RNAse free, Invitrogen) before cDNA synthesis, according to the manufacturer's instructions. First strand cDNA was then synthesized using Superscript II reverse transcriptase (Invitrogen) and a gene-specific primer mix with 2 pmol of each antisense oligonucleotide as described by the manufacturer. Genespecific oligonucleotide primers for $P$. falciparum genes $D X S, D X R, M C T, C M K, M C S$, GcpE, LytB, FPPs, PDXI, and liver stage antigen-1 (Lsa-1, negative control) were designed using the Primer Express Software v.1.5 (Applied Biosystems) (see Table I). PCR products from cDNA of each gene were cloned using the TOPO TA cloning kit (Invitrogen) and sequenced. The purified plasmids were quantified and linearized with PstI (Invitrogen). A representative standard curve was constructed for each gene using serial dilutions of the respective plasmids. For real-time PCR quantification of each generated cDNA, SYBR ${ }^{\circledR}$ green system was used $\left(\mathrm{SYBR}^{\circledR}\right.$ green PCR Master Mix, Applied Biosystems) on a GeneAmp ${ }^{\circledR}$ 5700 Sequence Detection system (Applied Biosystems) according to the manufacturer's instructions. Experimental PCR conditions included $300 \mathrm{nM}$ of each primer and cDNA corresponding to $100 \mathrm{ng}$ converted RNA in $25 \mu$ final volume per reaction. Each point was measured in triplicate. The real-time PCR consisted of one cycle of $50^{\circ} \mathrm{C}$ for $2 \mathrm{~min}$ and $95^{\circ} \mathrm{C}$ for $10 \mathrm{~min}$, followed by 40 cycles of $95^{\circ} \mathrm{C}$ for $15 \mathrm{~s}$ and $60^{\circ} \mathrm{C}$ for $1 \mathrm{~min}$. Am- plification specificity was checked using melting curve analysis following the manufacturer's instructions. Data analysis utilized the GeneAmp $5700{ }^{\circledR}$ SDS Software (version 1.3) to determinate the threshold cycle $\left(C_{t}\right)$ for each amplified product.

Cell extracts and high-performance liquid chromatography $(H P L C)$ - Each purified parasite stage was freeze-dried and successively extracted with hexane (3 $\times 0.5 \mathrm{ml})$ and ethanol/water $\left(1: 1, \mathrm{v} / \mathrm{v} ; 1 \times 1 \mathrm{ml}\right.$ at $55^{\circ} \mathrm{C}$ for $1.5 \mathrm{~h}$ ). Aliquots of each extract were monitored for radioactivity with a Beckman LS 5000 TD $\beta$-counter. Metabolic analyses by HPLC of DOXP, pyridoxal 5'phosphate (PNP), MEP, CDP-ME, CDP-MEP, MEcPP, dolichols and ubiquinones were carried out as described previously (Cassera et al. 2004).

\section{RESULTS}

Metabolic and transcript profiles of the MEP pathway during the intraerythrocytic cycle - The metabolite and transcript levels of the intermediates of the MEP pathway were analyzed to investigate the relationship between the intermediate levels and the metabolic requirement for the isoprenoid biosynthesis (dolichols and ubiquinones) throughout the intraerythrocytic cycle of $P$. falciparum (Fig. 2). Since GAP is also direct precursor in pyridoxal 5'-phosphate (PNP) biosynthesis (Fig. 1), PNP was included in our analyses (Burns et al. 2005, Gengenbacher et al. 2006, Zein et al. 2006). The metabolite levels of all analyzed intermediates showed an

TABLE I

Gene-specific oligonucleotides used for the real-time polymerase chain reaction transcript analysis

\begin{tabular}{|c|c|c|c|c|}
\hline Gene & PlasmoDB ID & Primers & $\begin{array}{l}\text { Amplicon } \\
\text { coordinates }\end{array}$ & $\begin{array}{l}\text { Length } \\
\text { (base pairs) }\end{array}$ \\
\hline DXS & MAL13P1.186 & $\begin{array}{l}\text { F: 5'-CTTTAAAGGTTATAATTGGAAGAAGTGGA-3' } \\
\text { R: 5'-GTGTTGCCCCATCCTCTCC-3' }\end{array}$ & $2768-2821$ & 3618 \\
\hline DXR & PF14_0641 & $\begin{array}{l}\text { F: 5'-AAAACCTTTAGATTTGGCTCAGGTT-3' } \\
\text { R: 5'-GTTGTTAGCTATTTCATTTGACGCA-3's }\end{array}$ & $1134-1284$ & 1467 \\
\hline MCT & PFA0340w & $\begin{array}{l}\text { F: 5'-ACTGAATTGATCGGTCCTAAGCA-3' } \\
\text { R: 5'-TTATACTGTGGATGATACATGAAAAATGTT-3' }\end{array}$ & $625-775$ & 2205 \\
\hline CMK & PFE0150c & $\begin{array}{l}\text { F: 5'-TGGCTCATCTAATGGTGCTACTG-3' } \\
\text { R: 5'-TCCGTACAATAAGCAAATCCAGAAC-3' }\end{array}$ & $876-1028$ & 1614 \\
\hline MCS & PFB0420w & $\begin{array}{l}\text { F: 5'-TTTTAGGTGCCTTAGGTTCTTTAGACAT-3' } \\
\text { R: 5'-ACATTAATATCCACGTTCCCAATATCA-3' }\end{array}$ & $386-536$ & 723 \\
\hline GcpE & PF10_0221 & $\begin{array}{l}\text { F: 5'-TGCTGACATTGTAAGGTTGACTGTT-3' } \\
\text { R: 5'-ACACATCAGCTGCCATTAAAGC-3' }\end{array}$ & $492-643$ & 2475 \\
\hline LytB & PFA0225w & $\begin{array}{l}\text { F: 5'-AATCAGAAATGTTCCAGCAGTATTACTT-3' } \\
\text { R: 5'-TGGAGGGTTTGTTAATAGGTTGACA-3' }\end{array}$ & $1356-1506$ & 1608 \\
\hline FPPs & PF11_0295 & $\begin{array}{l}\text { F: 5'-TGAGTGGGAAAAAGTGGCTTGTA-3' } \\
\text { R: 5'-CACCGCATTCTTAATTTCAACGT-3' }\end{array}$ & $192-342$ & 1110 \\
\hline PDX1 & MAL6P1.215 & $\begin{array}{l}\text { F: 5'-TTGTTAATTTTGCTGCTGGAGGT-3' } \\
\text { R: 5'-TTGCTAACAGCTGAAACGATTGA-3' }\end{array}$ & $626-776$ & 906 \\
\hline Lsa 1 & PF10_0356 & $\begin{array}{l}\text { F: 5'-CAGAAAATGAACGTGGATATTATATACCA-3' } \\
\text { R: 5'-TGTATATCCCTTCGTCCTTCAACAT-3' }\end{array}$ & $4055-4205$ & 4791 \\
\hline
\end{tabular}



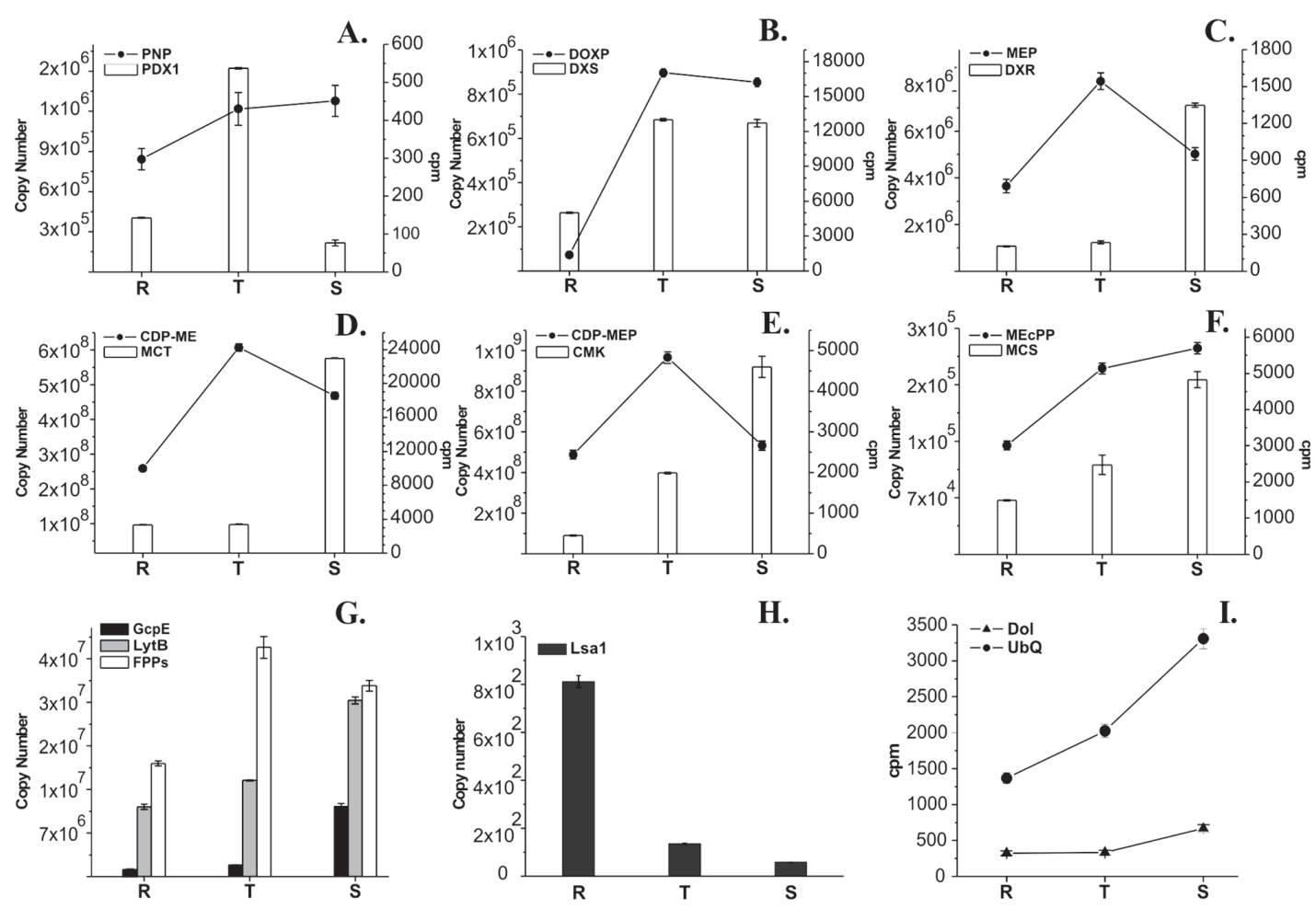

Fig. 2: metabolite and transcript profiles of each analyzed intermediate and its metabolizing enzyme during the intraerythrocytic cycle of Plasmodium falciparum. Panels A to F show the metabolite profile (line) and transcript level (bar) for the first five intermediates of the MEP pathway and PNP. Panels $\mathrm{G}$ and $\mathrm{H}$ display the transcript profiles for the GcpE, LytB, FPPs, and Lsa-1 genes. Dolichol and ubiquinone metabolite profiles are shown in panel I. Ring (R), trophozoite (T) and schizont (S). The absolute transcript quantities per $100 \mathrm{ng}$ converted RNA are indicated. Metabolite quantities are given in counts per minute $(\mathrm{cpm})$.

increase from the ring to the trophozoite stage (Fig. 2A$\mathrm{F}$, line). In the schizont stage, levels of PNP, DOXP, and MEcPP were maintained (Fig. 2A, B and F, line), but the levels of MEP, CDP-ME and CDP-MEP decreased relative to the trophozoite stage (Fig. 2C-E, line). Dolichol levels showed only a slight increase towards the schizont stage but the ubiquinone level was significantly increased from the ring to the schizont stage (Fig. 2I).

The intermediates HMBPP, IPP, DMAPP and geranyl pyrophosphate (GPP) were not resolved in the HPLC system used in our analyses. Nevertheless, the corresponding genes $G c p E, L y t B$, and $F P P s$ were included in the transcript analysis (Fig. 2G). Quantification and transcript analysis of these and the MEP pathway genes revealed different transcript profiles. Interestingly, except $D X S$, all genes involved in the MEP pathway (DXR, MCT, $C M K, M C S$, GcpE, and $L y t B$ ) had peak transcript abundance in the schizont stage (Fig. 2B-G, bar). DXS transcripts showed an increase in trophozoites and were maintained in schizont stage (Fig. 2B, bar). PDXI and FPPs transcript profiles displayed a maximum in trophozoite (Fig. $2 \mathrm{~A}$ and $\mathrm{G}$ bars). The $L s a-1$ gene is normally not expressed in the erythrocytic cycle and was employed as a negative control. Lsa-1 transcripts were almost undetectable in trophozoite and schizont stages and the quantity detected in ring stage was very low (Fig. $2 \mathrm{H}$ ), possibly due to relaxed transcription in ring stage (Blair et al. 2002).
Effect of fosmidomycin on the metabolic and transcript profiles - To evaluate if changes in the metabolic activity of the MEP pathway affect the transcript levels (Table II), we investigated the effect of fosmidomycin on metabolite and transcript levels. In general, the effect of fosmidomycin on the biosynthesis of the MEP pathway intermediates (DOXP to MEcPP), dolichols and ubiquinones was mainly observed in ring stages, where DOXP was accumulated, whereas MEP and downstream intermediates were observed in decreased levels. The most substantial effect on the end products of the isoprenoid biosynthesis was observed on the ubiquinones. Similar results, but less evident, were obtained in the schizont stage. The effect on the steady-state RNA levels of the analyzed genes was greater in the schizont stage, where the transcripts were accumulated, except MCT. A slight effect of fosmidomycin in the trophozoite stage was observed on metabolite and transcript levels. Interestingly, we observed that transcript level of $L y t B$, which codes for the last enzyme of the MEP pathway, was not altered. FPPs transcript levels were only affected in the schizont stage, where $30 \%$ increase of transcripts was detected in fosmidomycin-treated parasites. The level of the PDXI transcripts but not of the PNP metabolite was affected by fosmidomycin treatment in ring-stage parasites. In trophozoite and schizont stages both PNP metabolite and PDXI transcript levels were increased. 
TABLE II

Effect of $1 \mu \mathrm{M}$ of fosmidomycin on the metabolite and transcript levels of each analyzed intermediate and its respective metabolizing enzyme at the three characteristic developmental stages of Plasmodium falciparum

\begin{tabular}{|c|c|c|c|c|c|c|c|}
\hline \multirow[b]{2}{*}{ Intermediate } & \multicolumn{2}{|c|}{ Ring (\% effect) } & \multicolumn{2}{|c|}{ Trophozoite (\% effect) } & \multicolumn{2}{|c|}{ Schizont (\% effect) } & \multirow[b]{2}{*}{ Gene } \\
\hline & Metabolite & Transcript & Metabolite & Transcript & Metabolite & Transcript & \\
\hline PNP & 0 & -45 & 16 & 5 & 28 & 30 & PDXI \\
\hline DOXP & 4 & -19 & -2 & 0 & -23 & 50 & $D X S$ \\
\hline MEP & -13 & 0 & 0 & -17 & -15 & 16 & $D X R$ \\
\hline CDP-ME & -35 & 33 & -2 & 10 & -26 & -5 & $M C T$ \\
\hline CDP-MEP & -30 & 35 & 0 & -5 & -7 & 33 & $C M K$ \\
\hline $\mathrm{MEcPP}$ & -41 & 0 & -35 & 19 & 0 & 34 & $M C S$ \\
\hline НMBРP & - & 39 & - & -3 & - & 31 & $G c p E$ \\
\hline IPP / DMAPP & - & -7 & - & 0 & - & 0 & $L y t B$ \\
\hline GPP & - & 0 & - & 0 & - & 30 & FPPs \\
\hline Ubiquinones & -24 & - & -11 & - & -18 & - & - \\
\hline Dolichols & -2 & - & 0 & - & 0 & - & - \\
\hline
\end{tabular}

Positive values indicate increase of the intermediate or transcript levels and negative values indicate reduction of the intermediate or transcript levels compared to corresponding untreated control samples. A zero indicates no-effect. (-): not analyzed.

\section{DISCUSSION}

This is the first analysis that compares the effect of fosmidomycin on the metabolic and transcript profiles in P. falciparum, which has only the MEP pathway for IPP and DMAPP biosynthesis. A combined analysis of metabolic and transcript profiles may be a useful procedure for the identification of candidate enzymes as new drug targets (Urbanczyk-Wochniak et al. 2003).

Following the discovery of the MEP pathway, several studies have been conducted to understand the regulation of this metabolic route. Fruit development in the tomato has been widely used as a system for these studies due to the observation that a significant increase in the supply of isoprenoid precursors is required to permit the massive accumulation of carotenoids that takes place during ripening. In this process, MEP-pathway key enzyme transcripts are accumulated; meanwhile, the transcript levels of the other MEP enzymes remain unchanged (Lois et al. 2000, Rodriguez-Concepcion et al. 2001, 2003, Botella-Pavia et al. 2004). Despite the differences, $P$. falciparum intraerythrocytic development may be compared with tomato fruit ripening, since malaria parasites showed an increased biosynthesis of isoprenoids towards the end of schizogony (Fig. 2I). Therefore, using an approach similar to employed monitoring carotenoid synthesis during the fruit development in the tomato, we chose ubiquinones as the biological marker of isoprenoid precursor requirement from the MEP pathway in P. falciparum. Importantly, the comparative study of metabolite biosynthesis was conducted using the same absolute number of parasites, obtained from highly synchronized stages. In fact, ubiquinone levels were increased at the end of the intraerythrocytic cycle when the parasite is preparing for schizogony. Dolichol, the other isoprenoid studied herein, did not show significant variation in its levels during the intraerythrocytic cycle of the parasite, and its overall quantity detected was very low (Fig. 2I). This was expected because the main function of dolichols, as dolichyl phosphate, is their participation in glycoprotein synthesis in the endoplasmic reticulum, where the dolichol carrier is recycled (Chojnacki \& Dallner 1988, Burda \& Aebi 1999).

In this framework, the metabolite levels of most of the intermediates of the MEP pathway showed an increase in trophozoite and a decrease in schizont stages, when ubiquinones were accumulated. Interestingly, metabolic profiles showed that DOXP and CDP-ME were highly accumulated when compared to the other intermediates, mainly in the trophozoite and schizont stages (Fig. 2).

We considered that both DOXP and CDP-ME could act as a metabolite reserve, which might be used in the schizogony to sustain the high demand of isoprenoids, and both intermediates might be key metabolites of the MEP pathway in P. falciparum.

Metabolic results were correlated with the transcript profiles of genes involved in the MEP pathway. In general, transcript profiles of MEP pathway genes as well as PDXI and FPPs were similar to the results obtained by P. falciparum microarray studies (Le Roch et al. 2003, Llinas et al. 2006), taking in to consideration that the resolution of quantitative real time PCR is higher than the microarray technique. All genes involved in the MEP pathway had maximal transcript quantities in the schizont stage. Of those, $M C T$ and $C M K$ transcripts were always the most abundant in all stages (Fig. 2). Maximal transcript levels in the schizont stage were concomitant with an increase in the ubiquinone biosynthesis and a reduction of the respective MEP pathway intermediate. Several studies showed that in tomato fruit ripening the accumulation of carotenoids only requires increased levels of $D X S, D X R$, and LytB transcripts (Lois et al. 2000, RodriguezConcepcion et al. 2001, 2003, Botella-Pavia et al. 2004). Interestingly, we observed a positive correlation in $P$. falciparum between enhanced isoprenoid biosynthesis (ubiquinones) and accumulation of transcripts encoding all enzymes of the MEP pathway except for DXS. 
The effect of fosmidomycin treatment was stagedependent both at RNA transcripts and metabolite levels. Treatment with $1 \mu \mathrm{M}$ of fosmidomycin resulted, mainly in the ring stage, in a decrease in the intermediate biosynthesis of the MEP pathway as well as in the ubiquinone and dolichol biosynthesis as compared to controls. Slight differences were observed using asynchronous cultures in our previous work, predominantly in schizont stages (Cassera et al. 2004). In fosmidomycin-treated parasites, an increase of the transcript levels of the MEP pathway genes was mainly observed in the schizont stage. The general effect of fosmidomycin on the transcript levels of the analyzed genes was small (Table II). This effect is very similar to what was observed in previous studies when parasites were treated with pyrimethamine (Altschul et al. 1997, Gunasekera et al. 2003). These findings and our results reinforce the point of view that drug-target finding in P. falciparum is very difficult using only transcript-based analysis.

We observed a reduction of the level of PDXI transcripts by fosmidomycin treatment in ring-stage parasites but the PNP metabolite was not affected; while in trophozoite and schizont stages both PNP metabolite and $P D X 1$ transcript levels were increased. The substrates for $P$. falciparum PDX1 (ribose 5-phosphate and ribulose 5-phosphate and GAP as well as dihydroxyacetone) and its cytosolic localization have been recently characterized (Gengenbacher et al. 2006). The intermediate GAP is also substrate of DOXP synthase (Fig. 1). In $P$. falciparum, the MEP pathway occurs in the apicoplast, where the substrates for the DOXP synthesis are imported from the cytosol as phosphoenolpyruvate and dihydroxyacetone phosphate (DHAP). The DHAP is converted into GAP in a reversible reaction and can be transport outside of the apicoplast (Ralph et al. 2004 and references herein). Therefore, changes on the MEP pathway metabolites could affect the cytoplasmatic GAP and PNP levels.

Since the main effect of fosmidomycin treatment on the metabolic and transcript profiles was observed at different times during the developmental cycle and the effect on the transcript levels, we suggest a post-transcriptional regulation of the MEP pathway in $P$. falciparum.

\section{ACKNOWLEDGMENTS}

To Dr Silvano Wendel (Sírio Libanês Hospital, São Paulo) for the gift of erythrocytes; Dr D Arigoni (Laboratorium für Organische Chemie, Eidgenössische Technische Hochschule, Zürich, Switzerland) for a sample of 1-deoxy-D-xylulose and Dr H Jomaa (Biochemisches Institut der Justus-Liebig-Universität Gießen, Gießen, Germany) for the MEP pathway intermediates and fosmidomycin. To Drs HA del Portillo, C Winter, S Uliana, and V Schramm for stimulating discussions. To Dr A Sigari Moriscot for technical support in the real-time PCR analysis. To our laboratory colleagues for technical assistance.

\section{REFERENCES}

Altschul SF, Madden TL, Schaffer AA, Zhang J, Zhang Z, Miller W, Lipman DJ 1997. Gapped BLAST and PSI-BLAST: a new generation of protein database search programs. $\mathrm{Nu}$ cleic Acids Res 25: 3389-3402.

Barkovich R, Liao JC 2001. Metabolic engineering of isoprenoids. Metab Eng 3: 27-39.
Blair PL, Witney A, Haynes JD, Moch JK, Carucci DJ, Adams JH 2002. Transcripts of developmentally regulated Plasmodium falciparum genes quantified by real-time RT-PCR. Nucleic Acids Res 30: 2224-2231.

Borrmann S, Adegnika AA, Moussavou F, Oyakhirome S, Esser G, Matsiegui PB, Ramharter M, Lundgren I, Kombila M, Issifou S, Hutchinson D, Wiesner J, Jomaa H, Kremsner PG 2005. Short-course regimens of artesunate-fosmidomycin in treatment of uncomplicated Plasmodium falciparum malaria. Antimicrob Agents Chemother 49: 3749-3754.

Borrmann S, Lundgren I, Oyakhirome S, Impouma B, Matsiegui PB, Adegnika AA, Issifou S, Kun JF, Hutchinson D, Wiesner J, Jomaa H, Kremsner PG 2006. Fosmidomycin plus clindamycin for treatment of pediatric patients aged 1 to 14 years with Plasmodium falciparum malaria. Antimicrob Agents Chemother 50: 2713-2718.

Botella-Pavia P, Besumbes O, Phillips MA, Carretero-Paulet L, Boronat A, Rodriguez-Concepcion M 2004. Regulation of carotenoid biosynthesis in plants: evidence for a key role of hydroxymethylbutenyl diphosphate reductase in controlling the supply of plastidial isoprenoid precursors. Plant J 40: 188-199.

Bozdech Z, Llinas M, Pulliam BL, Wong ED, Zhu J, DeRisi JL 2003. The transcriptome of the intraerythrocytic developmental cycle of Plasmodium falciparum. PLoS Biol 1: E5.

Burda P, Aebi M 1999. The dolichol pathway of N-linked glycosylation. Biochim Biophys Acta 1426: 239-257.

Burns KE, Xiang Y, Kinsland CL, McLafferty FW, Begley TP 2005. Reconstitution and biochemical characterization of a new pyridoxal-5'-phosphate biosynthetic pathway. $J$ Am Chem Soc 127: 3682-3683.

Cassera MB, Gozzo FC, D’Alexandri FL, Merino EF, Del Portillo HA, Peres VJ, Almeida IC, Eberlin MN, Wunderlich G, Wiesner J, Jomaa H, Kimura EA, Katzin AM 2004. The methylerythritol phosphate pathway is functionally active in all intraerythrocytic stages of Plasmodium falciparum. $J$ Biol Chem 279: 51749-51759.

Chojnacki T, Dallner G 1988. The biological role of dolichol. Biochem J 251: 1-9.

Cranmer SL, Conant AR, Gutteridge WE, Halestrap AP 1995. Characterization of the enhanced transport of L- and D-lactate into human red blood cells infected with Plasmodium falciparum suggests the presence of a novel saturable lactate proton cotransporter. J Biol Chem 270: 15045-15052.

Eisenreich W, Bacher A, Arigoni D, Rohdich F 2004. Biosynthesis of isoprenoids via the non-mevalonate pathway. Cell Mol Life Sci 61: 1401-1426.

Elliott JL, Saliba KJ, Kirk K 2001. Transport of lactate and pyruvate in the intraerythrocytic malaria parasite, Plasmodium falciparum. Biochem J 355: 733-739.

Florens L, Washburn MP, Raine JD, Anthony RM, Grainger M, Haynes JD, Moch JK, Muster N, Sacci JB, Tabb DL, Witney AA, Wolters D, Wu Y, Gardner MJ, Holder AA, Sinden RE, Yates JR, Carucci DJ 2002. A proteomic view of the Plasmodium falciparum life cycle. Nature 419: 520-526.

Gengenbacher M, Fitzpatrick TB, Raschle T, Flicker K, Sinning I, Muller S, Macheroux P, Tews I, Kappes B 2006. Vitamin B6 biosynthesis by the malaria parasite Plasmodium falciparum: biochemical and structural insights. J Biol Chem 281: 3633-3641. 
Goldstein JL, Brown MS 1990. Regulation of the mevalonate pathway. Nature 343: 425-430.

Gunasekera AM, Patankar S, Schug J, Eisen G, Wirth DF 2003. Drug-induced alterations in gene expression of the asexual blood forms of Plasmodium falciparum. Mol Microbiol 50: 1229-1239.

Jomaa H, Wiesner J, Sanderbrand S, Altincicek B, Weidemeyer C, Hintz M, Turbachova I, Eberl M, Zeidler J, Lichtenthaler HK, Soldati D, Beck E 1999. Inhibitors of the nonmevalonate pathway of isoprenoid biosynthesis as antimalarial drugs. Science 285: 1573-1576.

Kimura EA, Couto AS, Peres VJ, Casal OL, Katzin AM 1996. $\mathrm{N}$-linked glycoproteins are related to schizogony of the intraerythrocytic stage in Plasmodium falciparum. J Biol Chem 271: 14452-14461.

Kuzuyama T, Shimizu T, Takahashi S, Seto H 1998. Fosmidomycin, a specific inhibitor of 1-deoxy-D-xylulose 5-phosphate reductoisomerase in the nonmevalonate pathway for terpenoid biosynthesis. Tetrahedron Lett 39: 7913-7916.

Lambros C, Vanderberg JP 1979. Synchronization of Plasmodium falciparum erythrocytic stages in culture. J Parasitol 65: 418-420.

Le Roch KG, Zhou Y, Blair PL, Grainger M, Moch JK, Haynes JD, De La Vega P, Holder AA, Batalov S, Carucci DJ, Winzeler EA 2003. Discovery of gene function by expression profiling of the malaria parasite life cycle. Science 301 : 1503-1508.

Lichtenthaler HK 1999. The 1-deoxy-D-xylulose-5-phosphate pathway of isoprenoid biosynthesis in plants. Annu Rev Plant Physiol Plant Mol Biol 50: 47-65.

Llinas M, Bozdech Z, Wong ED, Adai AT, DeRisi JL 2006. Comparative whole genome transcriptome analysis of three Plasmodium falciparum strains. Nucleic Acids Res 34: 11661173.

Lois LM, Rodriguez-Concepcion M, Gallego F, Campos N, Boronat A 2000. Carotenoid biosynthesis during tomato fruit development: regulatory role of 1-deoxy-D-xylulose 5-phosphate synthase. Plant J 22: 503-513.

Missinou MA, Borrmann S, Schindler A, Issifou S, Adegnika AA, Matsiegui PB, Binder R, Lell B, Wiesner J, Baranek T, Jomaa H, Kremsner PG 2002. Fosmidomycin for malaria. Lancet 360: 1941-1942.
Nirmalan N, Sims PF, Hyde JE 2004. Quantitative proteomics of the human malaria parasite Plasmodium falciparum and its application to studies of development and inhibition. Mol Microbiol 52: 1187-1199.

Ralph SA, van Dooren GG, Waller RF, Crawford MJ, Fraunholz MJ, Foth BJ, Tonkin CJ, Roos DS, McFadden GI 2004. Tropical infectious diseases: metabolic maps and functions of the Plasmodium falciparum apicoplast. Nat Rev Microbiol 2: 203-216.

Rodriguez-Concepcion M 2004. The MEP pathway: a new target for the development of herbicides, antibiotics and antimalarial drugs. Curr Pharm Des 10: 2391-2400.

Rodriguez-Concepcion M, Ahumada I, Diez-Juez E, Sauret-Gueto S, Lois LM, Gallego F, Carretero-Paulet L, Campos N, Boronat A 2001. 1-deoxy-D-xylulose 5-phosphate reductoisomerase and plastid isoprenoid biosynthesis during tomato fruit ripening. Plant J 27: 213-222.

Rodriguez-Concepcion M, Querol J, Lois LM, Imperial S, Boronat A 2003. Bioinformatic and molecular analysis of hydroxymethylbutenyl diphosphate synthase (GCPE) gene expression during carotenoid accumulation in ripening tomato fruit. Planta 217: 476-482.

Rohmer M 1999. The discovery of a mevalonate-independent pathway for isoprenoid biosynthesis in bacteria, algae and higher plants. Nat Prod Rep 16: 565-574.

Sacchettini JC, Poulter CD 1997. Creating isoprenoid diversity. Science 277: 1788-1789.

Sinensky M 2000. Recent advances in the study of prenylated proteins. Biochim Biophys Acta 1484: 93-106.

Snow RW, Guerra CA, Noor AM, Myint HY, Hay SI 2005. The global distribution of clinical episodes of Plasmodium falciparum malaria. Nature 434: 214-217.

Trager W, Jensen JB 1976. Human malaria parasites in continuous culture. Science 193: 673-675.

Urbanczyk-Wochniak E, Luedemann A, Kopka J, Selbig J, Roessner-Tunali U, Willmitzer L, Fernie AR 2003. Parallel analysis of transcript and metabolic profiles: a new approach in systems biology. EMBO Rep 4: 989-993.

Zein F, Zhang Y, Kang YN, Burns K, Begley TP, Ealick SE 2006. Structural insights into the mechanism of the PLP synthase holoenzyme from Thermotoga maritima. Biochemistry 45: 14609-14620. 
